\title{
On a conjecture of Lemmermeyer
}

\author{
S. AOUISSI, M. TALBI, M. C. ISMAILI and A. AZIZI
}

\begin{abstract}
Let $p \equiv 1(\bmod 3)$ be a prime and denote by $\zeta_{3}$ a primitive third root of unity. Recently, Lemmermeyer presented a conjecture about 3-class groups of pure cubic fields $L=\mathbb{Q}(\sqrt[3]{p})$ and of their normal closures $\mathrm{k}=\mathbb{Q}\left(\sqrt[3]{p}, \zeta_{3}\right)$. The purpose of this paper is to prove Lemmermeyer's conjecture.
\end{abstract}

Keywords: Pure cubic fields, Galois closure, 3-class groups, abelian type invariants.

Mathematics Subject Classification 2010: 11R11, 11R16, 11R20, 11R27, 11R29, $11 \mathrm{R} 37$.

\section{Introduction}

Let $L=\mathbb{Q}(\sqrt[3]{d})$ be a pure cubic field, where $d>1$ is a cubefree positive integer, $\mathrm{k}=\mathbb{Q}\left(\sqrt[3]{d}, \zeta_{3}\right)$ be its normal closure, and $C_{\mathrm{k}, 3}$ be the 3-component of the class group of $\mathrm{k}$.

In a collection of unsolved problems, Lemmermeyer proposed a conjecture for the special pure cubic field $\mathbb{Q}(\sqrt[3]{p})$, where $p \equiv 1(\bmod 3)$ is a prime number $[20$, Conjecture $5, \S 1.10$, p. 44]. This conjecture gives a necessary and sufficient condition for the 3 -class group $C_{\mathrm{k}, 3}$ to be isomorphic to either $\mathbb{Z} / 3 \mathbb{Z}$ or $\mathbb{Z} / 3 \mathbb{Z} \times \mathbb{Z} / 3 \mathbb{Z}$, and thus specifies the rank of $C_{\mathrm{k}, 3}$ as follows:

Conjecture 1.1. Let $L=\mathbb{Q}(\sqrt[3]{p})$ be a pure cubic field, where $p$ is a prime number such that $p \equiv 1(\bmod 3)$, and $\mathrm{k}=\mathbb{Q}\left(\sqrt[3]{p}, \zeta_{3}\right)$ be its normal closure. Let $C_{L, 3}$ (resp. $\left.C_{\mathrm{k}, 3}\right)$ be the 3 -component of the class group of $L$ (resp. k). Then:

1) $C_{L, 3}$ is a cyclic 3 -group, and if it contains a cyclic subgroup of order 9 , then $p \equiv 1(\bmod 9)$.

2) If $p \equiv 4,7(\bmod 9)$, then:

$$
C_{\mathrm{k}, 3} \simeq \begin{cases}\mathbb{Z} / 3 \mathbb{Z} & \text { if }\left(\frac{3}{p}\right)_{3} \neq 1 \\ (\mathbb{Z} / 3 \mathbb{Z})^{2} & \text { if }\left(\frac{3}{p}\right)_{3}=1 .\end{cases}
$$

3) If $p \equiv 1(\bmod 9)$, then $\operatorname{rank} C_{\mathrm{k}, 3} \in\{1,2\}$, independently of the value of $\left(\frac{3}{p}\right)_{3}$.

Here $(\dot{\bar{p}})_{3}$ is the cubic residue symbol.

In fact, Conjecture 1.1 for $p \equiv 4,7(\bmod 9)$ was first expressed in 1970 by Barrucand and Cohn [5, $\S 8$, p. 19], partially proved in 1976 by Barrucand, H. C. Williams and Baniuk [6, $\S 7$, Thm. 1, p. 321, and $\S 8$, Cnj. 1, p. 322], and mentioned again in 1982 by H. C. Williams [24, § 6, p. 273]. Conjecture 1.1 for $p \equiv 1(\bmod 9)$ was proved partially in 2005 by Gerth [12, Formulas p. 474, and Case 4, pp. 475-476], who also pointed out that Conjecture 1.1 for $p \equiv 4,7(\bmod 9)$ is still an open problem.

Based on results concerning the 3-class group $C_{\mathrm{k}, 3}$ in $\S 2.1$, we shall prove Conjecture 1.1 in $\S 2.2$. It will be underpinned by numerical examples obtained with the Computational Number Theory System PARI [23] in $\S 3.1$. Throughout this paper, we will use the following notations: 
- $p$ is a prime number such that $p \equiv 1(\bmod 3)$;

- $L=\mathbb{Q}(\sqrt[3]{d})$ is a pure cubic field, where $d>1$ is a cube-free positive integer;

- $\mathrm{k}_{0}=\mathbb{Q}\left(\zeta_{3}\right)$, where $\zeta_{3}=e^{2 i \pi / 3}$ denotes a primitive third root of unity;

- $\mathrm{k}=\mathbb{Q}\left(\sqrt[3]{d}, \zeta_{3}\right)$ is the normal closure of $L$;

- $\langle\tau\rangle=\operatorname{Gal}(\mathrm{k} / L)$ such that $\tau^{2}=i d, \tau\left(\zeta_{3}\right)=\zeta_{3}^{2}$ and $\tau(\sqrt[3]{d})=\sqrt[3]{d}$;

- $\langle\sigma\rangle=\operatorname{Gal}\left(\mathrm{k} / \mathrm{k}_{0}\right)$ such that $\sigma^{3}=i d, \sigma\left(\zeta_{3}\right)=\zeta_{3}, \sigma(\sqrt[3]{d})=\zeta_{3} \sqrt[3]{d}$ and $\tau \sigma=\sigma^{2} \tau$;

- $\lambda=1-\zeta_{3}$ and $\pi$ are prime elements of $\mathrm{k}_{0}$;

- $q^{*}=1$ or 0 according to whether $\zeta_{3}$ is or is not norm of an element of $\mathrm{k} \backslash\{0\}$;

- $u$ denotes the index of the subgroup $E_{0}$ generated by the units of intermediate fields of the extension $\mathrm{k} / \mathbb{Q}$ in the group of units of $\mathrm{k}$;

- $\mathcal{N}_{\mathrm{k} / \mathrm{k}_{0}}$ denotes the norm of $\mathrm{k}$ on $\mathrm{k}_{0}$;

- $t$ denotes the number of prime ideals ramified in $\mathrm{k} / \mathrm{k}_{0}$;

- $(\dot{\bar{p}})_{3}$ is the cubic residue symbol such that $\left(\frac{c}{p}\right)_{3}=1 \Leftrightarrow X^{3} \equiv c(\bmod p)$ has a solution in $\mathbb{Z} \Leftrightarrow c^{(p-1) / 3} \equiv 1(\bmod p)$, where $c \in \mathbb{Z}$, and $p$ is a prime number such that $p \nmid c$ and $p \equiv 1(\bmod 3)$;

- For an algebraic number field $F$ :

$-\mathcal{O}_{F}, E_{F}$ : the ring of integers and the group of units of $F$;

$-C_{F, 3}, \mathrm{~F}_{3}^{(1)}$ : the 3-class group and the Hilbert 3-class field of $F$;

- $[\mathcal{I}]$ : the class of a fractional ideal $\mathcal{I}$ in the class group of $F$;

\section{Proof of Conjecture 1.1}

\subsection{Preliminary results}

Let $d>1$ be a cubefree integer, and let $L=\mathbb{Q}(\sqrt[3]{d})$ be the pure cubic field with radicand $d$. We denote by $\zeta_{3}=(-1+i \sqrt{3}) / 2$ a primitive cube root of unity. By $\mathrm{k}_{0}$ we denote the third cyclotomic field $\mathbb{Q}\left(\zeta_{3}\right)$, by $\mathrm{k}=\mathbb{Q}\left(\sqrt[3]{d}, \zeta_{3}\right)$ the normal closure of the pure cubic field $L$, and by $C_{\mathrm{k}, 3}$ the 3-component of the class group of $\mathrm{k}$. Further, let $\langle\tau\rangle=\mathrm{Gal}(\mathrm{k} / L)$ and $\langle\sigma\rangle=\mathrm{Gal}\left(\mathrm{k} / \mathrm{k}_{0}\right)$.

The 3 -class group $C_{\mathrm{k}, 3}$ can be viewed as a $\mathbb{Z}_{3}\left[\zeta_{3}\right]$-module. According to $[11, \S 2$, Lemma 2.1 and Lemma 2.2, p. 53] we have:

$$
C_{\mathrm{k}, 3} \cong C_{\mathrm{k}, 3}^{+} \times C_{\mathrm{k}, 3}^{-} \text {and } C_{\mathrm{k}, 3}^{+} \cong C_{L, 3} .
$$

Define the 3-group $C_{\mathrm{k}, 3}^{(1-\sigma)^{i}}$ for each $i \in \mathbb{N}$ by $C_{\mathrm{k}, 3}^{(1-\sigma)^{i}}=\left\{\mathcal{A}^{(1-\sigma)^{i}} \mid \mathcal{A} \in C_{\mathrm{k}, 3}\right\}$. Since we always have $\left(1-\zeta_{3}\right)^{2} \cdot \mathbb{Z}_{3}\left[\zeta_{3}\right]=3 \cdot \mathbb{Z}_{3}\left[\zeta_{3}\right]$, then for each $i \in \mathbb{N}$ we have $C_{\mathrm{k}, 3}^{(1-\sigma)^{i+2}}=\left(C_{\mathrm{k}, 3}^{(1-\sigma)^{i}}\right)^{3}$. Consequently, we have the following equation for the rank of the group $C_{\mathrm{k}, 3}$ :

$$
\operatorname{rank} C_{\mathrm{k}, 3}=\operatorname{rank}\left(C_{\mathrm{k}, 3} / C_{\mathrm{k}, 3}^{3}\right)=\operatorname{rank}\left(C_{\mathrm{k}, 3} / C_{\mathrm{k}, 3}^{(1-\sigma)}\right)+\operatorname{rank}\left(C_{\mathrm{k}, 3}^{(1-\sigma)} / C_{\mathrm{k}, 3}^{(1-\sigma)^{2}}\right) .
$$

The fact that, for each $i \in \mathbb{N}, C_{\mathrm{k}, 3}^{(1-\sigma)^{i}} / C_{\mathrm{k}, 3}^{(1-\sigma)^{i+1}}$ is a $\mathbb{Z}_{3}[\langle\tau\rangle]$-module implies that:

$$
\operatorname{rank}\left(C_{\mathrm{k}, 3}^{(1-\sigma)^{i}} / C_{\mathrm{k}, 3}^{(1-\sigma)^{i+1}}\right)=\operatorname{rank}\left(C_{\mathrm{k}, 3}^{(1-\sigma)^{i}} / C_{\mathrm{k}, 3}^{(1-\sigma)^{i+1}}\right)^{+}+\operatorname{rank}\left(C_{\mathrm{k}, 3}^{(1-\sigma)^{i}} / C_{\mathrm{k}, 3}^{(1-\sigma)^{i+1}}\right)^{-} .
$$


So, for each $i \in \mathbb{N}$, we consider the homomorphism $\varphi_{i}$ as follows:

$$
\begin{aligned}
\varphi_{i}: C_{\mathrm{k}, 3}^{(1-\sigma)^{i}} / C_{\mathrm{k}, 3}^{(1-\sigma)^{i+1}} & \longrightarrow C_{\mathrm{k}, 3}^{(1-\sigma)^{i+1}} / C_{\mathrm{k}, 3}^{(1-\sigma)^{i+2}} \\
\mathcal{A} \bmod C_{\mathrm{k}, 3}^{(1-\sigma)^{i+1}} & \longmapsto \mathcal{A}^{1-\sigma} \bmod C_{\mathrm{k}, 3}^{(1-\sigma)^{i+2}}
\end{aligned}
$$

where $\mathcal{A} \in C_{\mathrm{k}, 3}^{(1-\sigma)^{i}}$. Since $C_{\mathrm{k}, 3}^{(1-\sigma)^{i}} / C_{\mathrm{k}, 3}^{(1-\sigma)^{i+1}}$ is an elementary abelian 3-group for each $i \in \mathbb{N}$, it can be viewed as vector space over $\mathbb{Z}_{3}$. Thus $\varphi_{i}$ is a surjective vector space homomorphism.

Let $\mathcal{B} \in C_{\mathrm{k}, 3}^{(1-\sigma)^{i}} / C_{\mathrm{k}, 3}^{(1-\sigma)^{i+1}}$, then:

$$
\left(\mathcal{B}^{1-\sigma}\right)^{\tau}=\left(\mathcal{B}^{\tau}\right)^{1-\sigma^{2}}=\mathcal{B}^{1-\sigma^{2}}=\mathcal{B}^{3-(1-\sigma)-\left(1+\sigma+\sigma^{2}\right)} \equiv\left(\mathcal{B}^{1-\sigma}\right)^{-1} \bmod C_{\mathrm{k}, 3}^{(1-\sigma)^{i+2}}
$$

if $\mathcal{B} \in\left(C_{\mathrm{k}, 3}^{(1-\sigma)^{i}} / C_{\mathrm{k}, 3}^{(1-\sigma)^{i+1}}\right)^{+}$, and

$$
\left(\mathcal{B}^{1-\sigma}\right)^{\tau}=\left(\mathcal{B}^{\tau}\right)^{1-\sigma^{2}}=\left(\mathcal{B}^{-1}\right)^{1-\sigma^{2}}=\left(\mathcal{B}^{-1}\right)^{3-(1-\sigma)-\left(1+\sigma+\sigma^{2}\right)} \equiv \mathcal{B}^{1-\sigma} \bmod C_{\mathrm{k}, 3}^{(1-\sigma)^{i+2}}
$$

if $\mathcal{B} \in\left(C_{\mathrm{k}, 3}^{(1-\sigma)^{i}} / C_{\mathrm{k}, 3}^{(1-\sigma)^{i+1}}\right)^{-}$. Thus for each $i$, the homomorphism $\varphi_{i} \operatorname{maps}\left(C_{\mathrm{k}, 3}^{(1-\sigma)^{i}} / C_{\mathrm{k}, 3}^{(1-\sigma)^{i+1}}\right)^{+}$ onto $\left(C_{\mathrm{k}, 3}^{(1-\sigma)^{i+1}} / C_{\mathrm{k}, 3}^{(1-\sigma)^{i+2}}\right)^{-}$and maps $\left(C_{\mathrm{k}, 3}^{(1-\sigma)^{i}} / C_{\mathrm{k}, 3}^{(1-\sigma)^{i+1}}\right)^{-}$onto $\left(C_{\mathrm{k}, 3}^{(1-\sigma)^{i+1}} / C_{\mathrm{k}, 3}^{(1-\sigma)^{i+2}}\right)^{+}$.

Put $q^{*}=0$ or 1 according to whether $\zeta_{3}$ is not or is norm of an element of $k \backslash\{0\}$. Let $t$ be the number of primes ramified in $\mathrm{k} / \mathrm{k}_{0}$ and $C_{\mathrm{k}, 3}^{(\sigma)}=\left\{\mathcal{A} \in C_{\mathrm{k}, 3} / \mathcal{A}^{\sigma}=\mathcal{A}\right\}$ be the ambiguous ideal class group of $\mathrm{k} / \mathrm{k}_{0}$, where $\sigma$ is a generator of $\mathrm{Gal}\left(\mathrm{k} / \mathrm{k}_{0}\right)$. Then, according to $[10, \S 5$, pp 91-92] we have

$$
\left|C_{\mathrm{k}, 3}^{(\sigma)}\right|=3^{t-2+q^{*}} \text {. }
$$

If we denote by $C_{\mathrm{k}_{0}, 3}$ the Sylow 3 -subgroup of the ideal class group of $\mathrm{k}_{0}$, then $C_{\mathrm{k}_{0}, 3}=\{1\}$, and by the exact sequence :

$$
1 \longrightarrow C_{\mathrm{k}, 3}^{(\sigma)} \longrightarrow C_{\mathrm{k}, 3} \stackrel{1-\sigma}{\longrightarrow} C_{\mathrm{k}, 3} \longrightarrow C_{\mathrm{k}, 3} / C_{\mathrm{k}, 3}^{1-\sigma} \longrightarrow 1
$$

we deduce that

$$
\left|C_{\mathrm{k}, 3}^{(\sigma)}\right|=\left|C_{\mathrm{k}, 3} / C_{\mathrm{k}, 3}^{1-\sigma}\right| .
$$

The fact that $C_{\mathrm{k}, 3}^{(\sigma)}$ and $C_{\mathrm{k}, 3} / C_{\mathrm{k}, 3}^{1-\sigma}$ are elementary abelian 3 -groups implies that:

$$
\operatorname{rank} C_{\mathrm{k}, 3}^{(\sigma)}=\operatorname{rank}\left(C_{\mathrm{k}, 3} / C_{\mathrm{k}, 3}^{1-\sigma}\right) .
$$

Next, we define the important index of subfield units for the normal closure of pure cubic fields as follows: Put $L^{\prime}=\mathbb{Q}\left(\zeta_{3} \sqrt[3]{d}\right)$ and $L^{\prime \prime}=\mathbb{Q}\left(\zeta_{3}^{2} \sqrt[3]{d}\right)$. Let $\mathcal{O}_{\mathrm{k}}, \mathcal{O}_{L}, \mathcal{O}_{L^{\prime}}, \mathcal{O}_{L^{\prime \prime}}$ and $\mathcal{O}_{\mathrm{k}_{0}}$, respectively, be the rings of integers of $\mathrm{k}, L, L^{\prime}, L^{\prime \prime}$ and $\mathrm{k}_{0}$. Let $E_{\mathrm{k}, 3}$ be the unit's group in $\mathcal{O}_{\mathrm{k}, 3}$, and let $E_{0}$ be the subgroup of $E_{\mathrm{k}, 3}$ generated by the units in the ring of integers $\mathcal{O}_{L}, \mathcal{O}_{L^{\prime}}, \mathcal{O}_{L^{\prime \prime}}$ and $\mathcal{O}_{\mathrm{k}_{0}}$. We let $u$ denote the index $\left[E_{\mathrm{k}, 3}: E_{0}\right]$. According to $[4, \S 12$, Theorem 12.1, p. 229], there are two possibilities, either $u=1$ or $u=3$.

To prove Conjecture 1.1, we must employ the following Lemmas:

Lemma 2.1. Let $L=\mathbb{Q}(\sqrt[3]{d})$ be a pure cubic field, where $d>1$ is a cubefree natural number, and $\mathrm{k}=\mathbb{Q}\left(\sqrt[3]{d}, \zeta_{3}\right)$ be its normal closure. Let $C_{L, 3}$ (resp. $\left.C_{\mathrm{k}, 3}\right)$ be the 3 -component of the class group of $L$ (resp. $\mathrm{k}), h_{L}$ the class number of $L$, and $u$ the index of subfield units, defined as above. Then:

1) $C_{\mathrm{k}, 3} \simeq \mathbb{Z} / 3 \mathbb{Z} \times \mathbb{Z} / 3 \mathbb{Z} \Leftrightarrow\left(3\right.$ divides $h_{L}$ exactly and $\left.u=3\right)$.

2) $C_{L, 3} \simeq C_{\mathrm{k}, 3} \simeq \mathbb{Z} / 3 \mathbb{Z} \Leftrightarrow\left(3\right.$ divides $h_{L}$ exactly and $\left.u=1\right)$. 
Proof.

1) If $C_{\mathrm{k}, 3} \simeq \mathbb{Z} / 3 \mathbb{Z} \times \mathbb{Z} / 3 \mathbb{Z}$, then $\left|C_{\mathrm{k}, 3}\right|=9$ and by $[4, \S 14$, Theorem 14.1, p. 232] we have $\left|C_{\mathrm{k}, 3}\right|=\frac{u}{3}\left|C_{L, 3}\right|^{2}=9$. We deduce that $u=3$ and $\left|C_{L, 3}\right|=3$, since the other value for $u$, namely 1 , is not possible because otherwise 27 would be a square.

Conversely, if 3 divides $h_{L}$ exactly and $u=3$, then $3^{2}$ divides the class number $h_{\mathrm{k}}$ of $\mathrm{k}$ exactly and $\left|C_{\mathrm{k}, 3}\right|=9$. By $\left[11, \S 2\right.$, Lemma 2.2, p. 53], the group $C_{\mathrm{k}, 3}^{+}$is cyclic of order 3 . On the other hand, by $\left[11, \S 2\right.$, Lemma 2.1, p. 53], $C_{\mathrm{k}, 3} \simeq C_{\mathrm{k}, 3}^{+} \times C_{\mathrm{k}, 3}^{-}$. Thus we have $\left|C_{\mathrm{k}, 3}\right|=\left|C_{\mathrm{k}, 3}^{+}\right| \cdot\left|C_{\mathrm{k}, 3}^{-}\right|=9$, so $\left|C_{\mathrm{k}, 3}^{-}\right|=3$ and $C_{\mathrm{k}, 3}^{-}$is also a cyclic group of order 3 . As $C_{\mathrm{k}, 3}$ is the direct product of two cyclic subgroups of order 3 , then $C_{\mathrm{k}, 3} \simeq \mathbb{Z} / 3 \mathbb{Z} \times \mathbb{Z} / 3 \mathbb{Z}$.

2) We have the same proof as above.

Lemma 2.2. Let $p$ be a prime number such that $p \equiv 1(\bmod 3)$. Let $L=\mathbb{Q}(\sqrt[3]{p})$, and $\mathrm{k}=$ $\mathbb{Q}\left(\sqrt[3]{p}, \zeta_{3}\right)$ be its normal closure. Then, $p=\pi_{1} \pi_{2}$, with $\pi_{1}$ and $\pi_{2}$ are two primes of $\mathrm{k}_{0}$ such that $\pi_{2}=\pi_{1}^{\tau}$ and $\pi_{1} \equiv \pi_{2} \equiv 1\left(\bmod 3 \mathcal{O}_{\mathrm{k}_{0}}\right)$, where $\langle\tau\rangle=\mathrm{Gal}(\mathrm{k} / L)$. Furthermore:

$$
\left[\left(\frac{\zeta_{3}}{\pi_{1}}\right)_{3}=\left(\frac{\zeta_{3}}{\pi_{2}}\right)_{3}=1\right] \Longleftrightarrow\left(\frac{\zeta_{3}}{p}\right)_{3}=1
$$

where $(\dot{\bar{p}})_{3}$ is the cubic residue symbol.

Proof. Let $p$ be a prime number such that $p \equiv 1(\bmod 3)$. Then according to $[17, \S 9$, Section 1, prop. 9.1.4, p.110] there is two primes $\pi_{1}$ and $\pi_{2}$ of $\mathrm{k}_{0}$ such that $p=\pi_{1} \pi_{2}, \pi_{2}=\pi_{1}^{\tau}$ and $\pi_{1} \equiv \pi_{2} \equiv 1\left(\bmod 3 \mathcal{O}_{\mathrm{k}_{0}}\right)$.

$\Leftarrow:$ It is clear that if $\left(\frac{\zeta_{3}}{p}\right)_{3}=1$ then $\left(\frac{\zeta_{3}}{\pi_{1}}\right)_{3}=\left(\frac{\zeta_{3}}{\pi_{2}}\right)_{3}=1$.

$\Rightarrow:$ If $\left(\frac{\zeta_{3}}{\pi_{1}}\right)_{3}=\left(\frac{\zeta_{3}}{\pi_{2}}\right)_{3}=1$, then the equations

$$
\left\{\begin{array}{l}
\zeta_{3} \equiv X^{3}\left(\bmod \pi_{1}\right) \\
\zeta_{3} \equiv Y^{3}\left(\bmod \pi_{2}\right)
\end{array}\right.
$$

are solvable in $\mathcal{O}_{\mathrm{k}_{0}}$, and we have $X \equiv Z\left(\bmod \pi_{1}\right)$ and $Y \equiv Z\left(\bmod \pi_{2}\right)$ because $\mathcal{O}_{\mathrm{k}_{0}} /\left(\pi_{1}\right)$ is a field. Then $\bar{X}=\bar{Z} \neq \overline{0}$ in $\mathcal{O}_{\mathrm{k}_{0}} /\left(\pi_{1}\right)$, then $\bar{X}^{3}=\bar{Z}^{3}$ in $\mathcal{O}_{\mathrm{k}_{0}} /\left(\pi_{1}\right)$, thus $X^{3} \equiv Z^{3}\left(\bmod \pi_{1}\right)$ is solvable in $\mathcal{O}_{\mathrm{k}_{0}}$. Similarly for $Y$, we obtain $Y^{3} \equiv Z^{3}\left(\bmod \pi_{2}\right)$ is solvable in $\mathcal{O}_{\mathrm{k}_{0}}$. Then

$$
\left\{\begin{array}{l}
\zeta_{3} \equiv Z^{3}\left(\bmod \pi_{1}\right) \\
\zeta_{3} \equiv Z^{3}\left(\bmod \pi_{2}\right)
\end{array}\right.
$$

are solvable in $\mathcal{O}_{\mathrm{k}_{0}}$. So $\left(\zeta_{3}-Z^{3}\right)$ is in $\mathcal{O}_{\mathrm{k}_{0}}$ because $\pi_{1}$ and $\pi_{2}$ are two different primes of $\mathrm{k}_{0}$. Since $\left(\pi_{1}, \pi_{2}\right)=1$, then according to Gauss's Theorem we get $\pi_{1} \pi_{2} \mid\left(\zeta_{3}-Z^{3}\right)$ in $\mathcal{O}_{\mathrm{k}_{0}}$, which implies that $\zeta_{3} \equiv Z^{3}(\bmod p)$ is solvable in $\mathcal{O}_{\mathrm{k}_{0}}$, since $p=\pi_{1} \pi_{2}$. Thus $\left(\frac{\zeta_{3}}{p}\right)_{3}=1$.

Lemma 2.3. Let $p$ be a prime number such that $p \equiv 1(\bmod 3)$. If $\left(\frac{\zeta_{3}}{p}\right)_{3}=1$, then $p \equiv$ $1(\bmod 9)$, where $(\dot{\bar{p}})_{3}$ is the cubic residue symbol.

Proof. Let $p$ be a prime number such that $p \equiv 1(\bmod 3)$. Let us assume $\left(\frac{\zeta_{3}}{p}\right)_{3}=1$.

Since $p \equiv 1(\bmod 3)$, then according to $\left[17, \S 9\right.$, Section 1 , prop. 9.1.4, p.110], $p=\pi_{1} \pi_{2}$ where $\pi_{1}$ and $\pi_{2}$ are two primes of $\mathrm{k}_{0}$ such that $\pi_{2}=\pi_{1}^{\tau}$. According to $[19, \S \S 7.3$, Theorem 7.8, p. 217] 
we obtain $\pi_{1}=a+b \zeta_{3}$ and $\pi_{2}=a+b \zeta_{3}^{2}$, with $a=3 m+1$ and $b=3 n$, where $(n, m) \in \mathbb{N}^{2}$. Then:

$$
\begin{aligned}
p & =\pi_{1} \pi_{2} \\
& =\left(a+b \zeta_{3}\right)\left(a+b \zeta_{3}^{2}\right) \\
& =a^{2}+b^{2}-a b \\
& =(3 m+1)^{2}+(3 n)^{2}-(3 m+1)(3 n) \\
& =9 m^{2}+9 n^{2}-9 m n+6 m-3 n+1 \\
& \equiv 6 m-3 n+1 \quad(\bmod 9)
\end{aligned}
$$

According to [19, § $§ 7.3$, Theorem 7.8, p. 217] we have

$$
\left(\frac{\zeta_{3}}{p}\right)_{3}=\zeta_{3}^{\frac{1-a-b}{3}}=\zeta_{3}^{-(m+n)}
$$

then

$$
\left(\frac{\zeta_{3}}{p}\right)_{3}=\left\{\begin{array}{lcc}
1, & \text { if } m+n \equiv 0 \quad(\bmod 3) \\
\zeta_{3}, & \text { if } m+n \equiv-1 \quad(\bmod 3) \\
\zeta_{3}^{2}, & \text { if } m+n \equiv-2 \quad(\bmod 3)
\end{array}\right.
$$

since $\left(\frac{\zeta_{3}}{p}\right)_{3}=1$, then there exist $k \in \mathbb{N}$ such that $m+n=3 k$. So

$$
\begin{aligned}
p & \equiv 6 m-3 n+1 \quad(\bmod 9) \\
& \equiv 6 m-3(3 k-m)+1 \quad(\bmod 9) \\
& \equiv 9 m-9 k+1 \quad(\bmod 9) \\
& \equiv 1 \quad(\bmod 9) .
\end{aligned}
$$

Lemma 2.4. Let $\mathrm{k}=\mathbb{Q}\left(\sqrt[3]{p}, \zeta_{3}\right)$, where $p$ is a prime number such that $p \equiv 1(\bmod 3)$. Let $C_{\mathrm{k}, 3}^{(\sigma)}$ be the ambiguous ideal class group of $\mathrm{k} / \mathrm{k}_{0}$, where $\sigma$ is a generator of $\operatorname{Gal}\left(\mathrm{k} / \mathrm{k}_{0}\right)$. Then $\left|C_{\mathrm{k}, 3}^{(\sigma)}\right|=3$.

Proof. Since $p \equiv 1(\bmod 3)$, then according to $[17, \S 9$, Section 1, prop. 9.1.4, p.110] we have $p=\pi_{1} \pi_{2}$, where $\pi_{1}$ and $\pi_{2}$ are two primes of $\mathrm{k}_{0}$ such that $\pi_{2}=\pi_{1}^{\tau}$ and $\pi_{1} \equiv \pi_{2} \equiv 1\left(\bmod 3 \mathcal{O}_{\mathrm{k}_{0}}\right)$. We study all cases depending on the congruence class of $p$ modulo 9 , then:

- If $p \equiv 4$ or $7(\bmod 9)$, then according to $[9, \S 4$, pp. $51-55]$, the prime 3 is ramified in the field $L$, so the prime ideal $\left(1-\zeta_{3}\right)$ is ramified in $\mathrm{k} / \mathrm{k}_{0}$. Also $\pi_{1}$ and $\pi_{2}$ are totally ramified in $\mathrm{k}$. So $t=3$. As $p \equiv 4$ or $7(\bmod 9)$, then $\pi_{1} \pi_{2}=p \equiv 4$ or $7\left(\bmod \left(1-\zeta_{3}\right)^{4}\right)$ because $9=3^{2}=\left(-\zeta_{3}^{2}\left(1-\zeta_{3}\right)^{2}\right)^{2}=\zeta_{3}\left(1-\zeta_{3}\right)^{4}$ (cf. [17, § 9, Section 1, prop. 9.1.4, p.110]), so $p=\pi_{1} \pi_{2} \equiv 4$ or $7\left(\bmod \left(1-\zeta_{3}\right)^{3}\right)$. Thus $\pi_{1}$ and $\pi_{2} \not \equiv 1\left(\bmod \left(1-\zeta_{3}\right)^{3}\right)$, and according to $[10, \S 5$, pp. 91-92] we obtain

$$
\left(\frac{\zeta_{3}, p}{p}\right)_{3} \neq 1
$$

where the symbol $(\stackrel{-}{-})_{3}$ is the cubic Hilbert symbol. We deduce that $\zeta_{3}$ is not a norm in the extension $\mathrm{k} / \mathrm{k}_{0}$, so $q^{*}=0$. By Equation (2) we get $\left|C_{\mathrm{k}, 3}^{(\sigma)}\right|=3$.

- If $p \equiv 1(\bmod 9)$, then the prime ideals which ramify in $\mathrm{k} / \mathrm{k}_{0}$ are $\left(\pi_{1}\right)$ and $\left(\pi_{2}\right)$, so $t=2$. Moreover, $\pi_{1} \equiv \pi_{2} \equiv 1\left(\bmod \left(1-\zeta_{3}\right)^{3}\right)$. Thus, according to [10, $\S 5$, pp. 91-92], the cubic Hilbert symbol is:

$$
\left(\frac{\zeta_{3}, p}{\pi_{1}}\right)_{3}=\left(\frac{\zeta_{3}, p}{\pi_{2}}\right)_{3}=1
$$

We conclude that $\zeta_{3}$ is a norm in the extension $\mathrm{k} / \mathrm{k}_{0}$, that is, $q^{*}=1$, so according to Equation (2) we obtain $\left|C_{\mathrm{k}, 3}^{(\sigma)}\right|=3$. 
Next, we specify the rank of $C_{\mathrm{k}, 3}$ as follows:

Lemma 2.5. Let $\mathrm{k}=\mathbb{Q}\left(\sqrt[3]{p}, \zeta_{3}\right)$, where $p$ is a prime number such that $p \equiv 1(\bmod 3)$. Let $s$ be the non-null positive integer such that $C_{\mathrm{k}, 3}^{(\sigma)} \subseteq C_{\mathrm{k}, 3}^{(1-\sigma)^{s-1}}$ but $C_{\mathrm{k}, 3}^{(\sigma)} \nsubseteq C_{\mathrm{k}, 3}^{(1-\sigma)^{s}}$, where $C_{\mathrm{k}, 3}^{(\sigma)}$ denotes the ambiguous ideal class group of $\mathrm{k} / \mathbb{Q}\left(\zeta_{3}\right)$ and $\sigma$ is a generator of $\mathrm{Gal}\left(\mathrm{k} / \mathbb{Q}\left(\zeta_{3}\right)\right)$. Let $C_{\mathrm{k}, 3}$ be the 3 -component of the class group of $\mathrm{k}$, then $\left|C_{\mathrm{k}, 3}\right|=3^{s}$, and rank $C_{\mathrm{k}, 3}=1$ or 2 . Furthermore

$$
C_{\mathrm{k}, 3} \simeq \begin{cases}\left(\mathbb{Z} / 3^{\frac{s}{2}} \mathbb{Z}\right)^{2}, & \text { if } s \text { is even }, \\ \mathbb{Z} / 3^{\frac{s+1}{2}} \mathbb{Z} \times \mathbb{Z} / 3^{\frac{s-1}{2}} \mathbb{Z}, & \text { if } s \text { is odd },\end{cases}
$$

Proof. We have $p \equiv 1(\bmod 3)$, then by Lemma $2.4,\left|C_{\mathrm{k}, 3}^{(\sigma)}\right|=3$. Since $\left|C_{\mathrm{k}, 3}^{(\sigma)}\right|=\left|C_{\mathrm{k}, 3} / C_{\mathrm{k}, 3}^{1-\sigma}\right|$, then

$$
\left|C_{\mathrm{k}, 3} / C_{\mathrm{k}, 3}^{1-\sigma}\right|=\left|C_{\mathrm{k}, 3}^{1-\sigma} / C_{\mathrm{k}, 3}^{(1-\sigma)^{2}}\right|=\ldots=\left|C_{\mathrm{k}, 3}^{(1-\sigma)^{s-1}} / C_{\mathrm{k}, 3}^{(1-\sigma)^{s}}\right|=3,
$$

where $s$ is the positive integer defined above, and we have

$$
\left|C_{\mathrm{k}, 3}\right|=\left|C_{\mathrm{k}, 3} / C_{\mathrm{k}, 3}^{1-\sigma}\right| \times\left|C_{\mathrm{k}, 3}^{1-\sigma} / C_{\mathrm{k}, 3}^{(1-\sigma)^{2}}\right| \times \ldots \times\left|C_{\mathrm{k}, 3}^{(1-\sigma)^{s-1}} / C_{\mathrm{k}, 3}^{(1-\sigma)^{s}}\right|=3^{s} .
$$

According to Equation (1), it is easy to see that

$$
\operatorname{rank} C_{\mathrm{k}, 3}= \begin{cases}1 & \text { if } s=1 \\ 2 & \text { if } s>1\end{cases}
$$

Next, let $\mathrm{k}_{3}^{(1)}$ be the maximal abelian unramified 3-extension of $\mathrm{k}$. So $\mathrm{k}_{3}^{(1)} / \mathrm{k}_{0}$ is Galois, and according to class field theory we have

$$
\operatorname{Gal}\left(\mathrm{k}_{3}^{(1)} / \mathrm{k}\right) \cong C_{\mathrm{k}, 3}
$$

We denote by $\left(\mathrm{k} / \mathrm{k}_{0}\right)^{*}$ the maximal abelian extension of $\mathrm{k}_{0}$ contained in $\mathrm{k}_{3}^{(1)}$, which is called the relative genus field of $\mathrm{k} / \mathrm{k}_{0}$ (cf. [15, § 2, p. VII-3]). So the commutator subgroup of Gal $\left(\mathrm{k}_{3}^{(1)} / \mathrm{k}_{0}\right)$ coincides with $\mathrm{Gal}\left(\mathrm{k}_{3}^{(1)} /\left(\mathrm{k} / \mathrm{k}_{0}\right)^{*}\right)$ and thus

$$
\operatorname{Gal}\left(\left(\mathrm{k} / \mathrm{k}_{0}\right)^{*} / \mathrm{k}_{0}\right) \cong \operatorname{Gal}\left(\mathrm{k}_{3}^{(1)} / \mathrm{k}_{0}\right) / \operatorname{Gal}\left(\mathrm{k}_{3}^{(1)} /\left(\mathrm{k} / \mathrm{k}_{0}\right)^{*}\right)
$$

The fact that $\mathrm{k} / \mathrm{k}_{0}$ is abelian and that $\mathrm{k} \subseteq\left(\mathrm{k} / \mathrm{k}_{0}\right)^{*}$ implies that Gal $\left(\mathrm{k}_{3}^{(1)} /\left(\mathrm{k} / \mathrm{k}_{0}\right)^{*}\right)$ coincides with $C_{\mathrm{k}, 3}^{1-\sigma}$, with the aid of the isomorphism (3) above and by Artin's reciprocity law. $C_{\mathrm{k}, 3}^{1-\sigma}$ is called the principal genus of $C_{\mathrm{k}, 3}$. Thus

$$
\operatorname{Gal}\left(\left(\mathrm{k} / \mathrm{k}_{0}\right)^{*} / \mathrm{k}\right) \cong C_{\mathrm{k}, 3} / C_{\mathrm{k}, 3}^{1-\sigma}
$$

Since $\left|C_{\mathrm{k}, 3} / C_{\mathrm{k}, 3}^{1-\sigma}\right|=\left|C_{\mathrm{k}, 3}^{(\sigma)}\right|=3$, then $\left(\mathrm{k} / \mathrm{k}_{0}\right)^{*}$ is an unramified cyclic cubic extension of $\mathrm{k}$ of degree 3 which is an abelian extension of $\mathrm{k}_{0}$.

Since $p \equiv 1(\bmod 3)$, then according to $\left[17, \S 9\right.$, Section 1 , prop. 9.1.4, p.110] we have $p=\pi_{1} \pi_{2}$, where $\pi_{1}$ and $\pi_{2}$ are two primes in $\mathrm{k}_{0}$ such that $\pi_{2}=\pi_{1}^{\tau}$. Let $\zeta_{p}$ be a primitive $p$ th root of unity. If we denote by $\mathrm{k}_{p}$ the unique sub-field of $\mathbb{Q}\left(\zeta_{p}\right)$ of degree 3 in which only $p$ ramifies, then $\mathrm{k}_{p} \mathrm{k}_{0}=\mathbb{Q}\left(\zeta_{3}, \sqrt[3]{\pi_{1} \pi_{2}^{2}}\right)$. Hence $\left(\mathrm{k} / \mathrm{k}_{0}\right)^{*}=\mathrm{k}\left(\sqrt[3]{\pi_{1} \pi_{2}^{2}}\right)$. From the congruence:

$$
\left(\pi_{1} \pi_{2}^{2}\right)^{\tau}=\pi_{2} \pi_{1}^{2} \equiv\left(\pi_{1} \pi_{2}^{2}\right)^{-1} \bmod \left(\mathrm{k}^{\times}\right)^{3}
$$

we conclude by Kummer theory and according to [11, Proposition 2.4, p. 54] that: 


$$
\left|\left(C_{\mathrm{k}, 3} / C_{\mathrm{k}, 3}^{1-\sigma}\right)^{+}\right|=3 \text { and }\left|\left(C_{\mathrm{k}, 3} / C_{\mathrm{k}, 3}^{1-\sigma}\right)^{-}\right|=1 .
$$

However, by the observations on the surjective maps $\varphi_{i}$ above, for each integer $i$ such that $0 \leq$ $i \leq s-1$ we have:

$$
\left|\left(C_{\mathrm{k}, 3}^{(1-\sigma)^{i}} / C_{\mathrm{k}, 3}^{(1-\sigma)^{i+1}}\right)^{+}\right|= \begin{cases}3, & \text { if } s \text { is even, } \\ 1, & \text { if } s \text { is odd }\end{cases}
$$

and

$$
\left|\left(C_{\mathrm{k}, 3}^{(1-\sigma)^{i}} / C_{\mathrm{k}, 3}^{(1-\sigma)^{i+1}}\right)^{-}\right|= \begin{cases}1, & \text { if } s \text { is even, } \\ 3, & \text { if } s \text { is odd }\end{cases}
$$

We conclude that:

$$
\left|C_{\mathrm{k}, 3}^{+}\right|=\left\{\begin{array}{l}
3^{\frac{s}{2}}, \quad \text { if } s \text { is even } \\
3^{\frac{s+1}{2}}, \quad \text { if } s \text { is odd }
\end{array}\right.
$$

and

$$
\left|C_{\mathrm{k}, 3}^{-}\right|=\left\{\begin{array}{l}
3^{\frac{s}{2}}, \quad \text { if } s \text { is even, } \\
3^{\frac{s-1}{2}}, \quad \text { if } s \text { is odd. }
\end{array}\right.
$$

Since rank $C_{\mathrm{k}, 3} \in\{1,2\}$, then $C_{\mathrm{k}, 3}^{+}$and $C_{\mathrm{k}, 3}^{-}$are a cyclic 3 -groups. Hence:

$$
C_{\mathrm{k}, 3} \simeq \begin{cases}\left(\mathbb{Z} / 3^{\frac{s}{2}} \mathbb{Z}\right)^{2}, & \text { if } s \text { is even }, \\ \mathbb{Z} / 3^{\frac{s+1}{2}} \mathbb{Z} \times \mathbb{Z} / 3^{\frac{s-1}{2}} \mathbb{Z}, & \text { if } s \text { is odd }\end{cases}
$$

Lemma 2.6. Let $\mathrm{k}=\mathbb{Q}\left(\sqrt[3]{p}, \zeta_{3}\right)$, where $p$ is a prime number such that $p \equiv 1(\bmod 3)$. Let $C_{\mathrm{k}, 3}^{(\sigma)}=\left\{\mathcal{A} \in C_{\mathrm{k}, 3} / \mathcal{A}^{\sigma}=\mathcal{A}\right\}$ be the ambiguous ideal class group of $\mathrm{k} / \mathrm{k}_{0}$, and $u$ be the index of subfield units defined as above.

(i) If $\left|\left(C_{\mathrm{k}, 3}^{(\sigma)}\right)^{+}\right|=3$, then $u=1$,

(ii) If $\left|\left(C_{\mathrm{k}, 3}^{(\sigma)}\right)^{+}\right|=1$, then $u=3$,

where $\left(C_{\mathrm{k}, 3}^{(\sigma)}\right)^{+}$and $\left(C_{\mathrm{k}, 3}^{(\sigma)}\right)^{-}$are defined in [11, § 2, Lemma 2.1, p. 53].

Proof. Let $C_{\mathrm{k}, 3}$ be the 3-component of the class group of k. According to [11, § 2, Lemma 2.1 and Lemma 2.2, p. 53], $C_{\mathrm{k}, 3} \cong C_{L, 3} \times C_{\mathrm{k}, 3}^{-}$, where $C_{L, 3}$ is the 3-component of the class group of $L=\mathbb{Q}(\sqrt[3]{p})$. Let $s$ be the positive integer defined in Lemma 2.5. From proof of Lemma 2.5 we obtain:

$$
\left|C_{L, 3}\right|= \begin{cases}3^{\frac{s}{2}}, & \text { if } s \text { is even, } \\ 3^{\frac{s+1}{2}}, & \text { if } s \text { is odd, }\end{cases}
$$

and

$$
\left|C_{\mathrm{k}, 3}^{-}\right|=\left\{\begin{array}{l}
3^{\frac{s}{2}}, \text { if } s \text { is even, } \\
3^{\frac{s-1}{2}}, \quad \text { if } s \text { is odd. }
\end{array}\right.
$$

According to $\left[4, \S 14\right.$, Theorem 14.1, p. 232], $\left|C_{\mathrm{k}, 3}\right|=\frac{u}{3}\left|C_{L, 3}\right|^{2}$, where $u=1$ or 3 . By Lemma 2.5, we have $\left|C_{\mathrm{k}, 3}\right|=3^{s}$, thus:

(i) If $\left|\left(C_{\mathrm{k}, 3}^{(\sigma)}\right)^{+}\right|=3$ and $\left|\left(C_{\mathrm{k}, 3}^{(\sigma)}\right)^{-}\right|=1$, the integer $s$ is odd, whence $\left|C_{\mathrm{k}, 3}\right|$ is not a square, and thus $u=1$. 
(ii) Conversely, if $\left|\left(C_{\mathrm{k}, 3}^{(\sigma)}\right)^{+}\right|=1$ and $\left|\left(C_{\mathrm{k}, 3}^{(\sigma)}\right)^{-}\right|=3$, the integer $s$ is even, whence $\left|C_{\mathrm{k}, 3}\right|$ is a square, and thus $u=3$.

Lemma 2.7. Let $L=\mathbb{Q}(\sqrt[3]{p})$, where $p$ is a prime number such that $p \equiv 1(\bmod 3)$, and $\mathrm{k}=$ $\mathbb{Q}\left(\sqrt[3]{p}, \zeta_{3}\right)$. The prime $p$ decomposes in the field $\mathrm{k}$ as $\mathcal{P}^{3} \mathcal{Q}^{3}$, where $\mathcal{P}$ and $\mathcal{Q}$ are two prime ideals of $\mathrm{k}$, and $\mathcal{Q}$ is the conjugate of $\mathcal{P}$. Let $u$ and $s$ be the integers defined as above. Then the following statements are equivalent:

1) $u=3$;

2) $s$ is even;

3) $\mathcal{P}$ is not principal.

Proof. Let $C_{\mathrm{k}, 3}^{(\sigma)}$ be the group of ambiguous ideal classes of $\mathrm{k} / \mathrm{k}_{0}$. Since $p \equiv 1(\bmod 3)$, then by Lemma 2.4, $\left|C_{k, 3}^{(\sigma)}\right|=3$. If we denote by $I_{\mathrm{k}}$ the group of fractional ideals of $\mathrm{k}$, we let $S_{\mathrm{k}, 3}^{(\sigma)}=$ $\left\{\mathcal{A} \in C_{\mathrm{k}, 3} \mid \exists \mathcal{B} \in I_{\mathrm{k}}\right.$ such that $\mathcal{A}=[\mathcal{B}]$ and $\left.\mathcal{B}^{1-\sigma}=(1)\right\}$ be the group of strongly ambiguous ideal classes of the cyclic extension $\mathrm{k} / \mathrm{k}_{0}$. It is known that $S_{\mathrm{k}, 3}^{(\sigma)}$ is generated by the ideal classes of the primes ramified in $\mathrm{k} / \mathrm{k}_{0}$.

$1) \Rightarrow 2)$ : Let $h_{L, 3}\left(\right.$ resp. $\left.h_{\mathrm{k}, 3}\right)$ be the 3 -class number of $L$ (resp k). By $[4, \S 14$, Theorem 14.1, p. 232] we have $h_{\mathrm{k}, 3}=\frac{u}{3} h_{L, 3}^{2}$. If $u=3$, then $h_{\mathrm{k}, 3}$ is a square, so the integer $s$ is even.

$2) \Rightarrow 3):$ First, since $p \equiv 1(\bmod 3)$, then by $\left[17, \S 9\right.$, Section 1 , prop. 9.1.4, p.110] we have $p=\pi_{1} \pi_{2}$, where $\pi_{1}$ and $\pi_{2}$ are two primes of $\mathrm{k}_{0}$ such that $\pi_{1}=\pi_{2}^{\tau}$ and $\pi_{2}=\pi_{1}^{\tau}$ and $\pi_{1} \equiv \pi_{2} \equiv$ $1\left(\bmod 3 \mathcal{O}_{\mathrm{k}_{0}}\right)$. Furthermore, $\pi_{1}$ and $\pi_{2}$ are ramified in $\mathrm{k} / \mathrm{k}_{0}$, so there exist two prime ideals $\mathcal{P}$ and $\mathcal{Q}$ of $\mathrm{k}$ such that $\pi_{1} \mathcal{O}_{\mathrm{k}}=\mathcal{P}^{3}$ and $\pi_{2} \mathcal{O}_{\mathrm{k}}=\mathcal{Q}^{3}$. Then $p \mathcal{O}_{\mathrm{k}}=\mathcal{P}^{3} \mathcal{Q}^{3}$.

Next, let $s$ be even, where $s$ is the non-null positive integer defined in Lemma 2.5. We shall prove that the prime ideal $\mathcal{P}$ is not principal. Assume that $\mathcal{P}$ is principal. The fact that $p \equiv 1(\bmod 3)$ implies that $\left|C_{\mathrm{k}, 3}^{(\sigma)}\right|=3$ according to Lemma 2.4. We study all cases in dependence on the congruence class of $p$ modulo 9:

- If $p \equiv 1(\bmod 9)$, then 3 is decomposes in $L$ by $[9, \S 4$, pp. 51-55]. The prime ideals which ramify in $\mathrm{k} / \mathrm{k}_{0}$ are $\left(\pi_{1}\right)$ and $\left(\pi_{2}\right)$. Since $\mathcal{P}$ is principal, the prime ideal $\mathcal{Q}$ is also principal. Thus a generator of $C_{\mathrm{k}, 3}^{(\sigma)}$ does not contain the classes of prime ideals lying above the primes ramified in the extension $\mathrm{k} / \mathrm{k}_{0}$. So, a generator of $C_{\mathrm{k}, 3}^{(\sigma)}$ comes from $C_{\mathrm{k}, 3}^{+}$(cf. [13, Proposition 2, part (1), p. 679]). This implies that

$$
\left|\left(C_{\mathrm{k}, 3}^{(\sigma)}\right)^{+}\right|=3 \text { and }\left|\left(C_{\mathrm{k}, 3}^{(\sigma)}\right)^{-}\right|=1,
$$

where $\left(C_{\mathrm{k}, 3}^{(\sigma)}\right)^{+}$and $\left(C_{\mathrm{k}, 3}^{(\sigma)}\right)^{-}$are defined in [11, § 2, Lemma 2.1, p. 53]. By Lemma 2.6 we see that the integer $s$ is odd. This contradicts the fact that $s$ is even. Thus $\mathcal{P}$ is not principal.

- If $p \equiv 4$ or $7(\bmod 9)$, then 3 is ramified in $L$ by $[9, \S 4$, pp. 51-55], so the prime ideals which ramify in $\mathrm{k} / \mathrm{k}_{0}$ are $\left(1-\zeta_{3}\right),\left(\pi_{1}\right)$ and $\left(\pi_{2}\right)$. Let $I$ be the unique prime ideal above $\left(1-\zeta_{3}\right)$ in $\mathrm{k}$. The fact that $p \equiv 4$ or $7(\bmod 9)$ implies according to $[1, \S 3$, Lemma 3.1 , p. 16] that $\pi_{i} \not \equiv 1\left(\bmod \left(1-\zeta_{3}\right)^{3}\right)$ for $i=\{1,2\}$. Thus, according to $[1, \S 3$, Lemma 3.3 , p. 17] we get $\zeta_{3}$ is not a norm in the extension k $/ \mathrm{k}_{0}$, so $S_{\mathrm{k}, 3}^{(\sigma)}=C_{\mathrm{k}, 3}^{(\sigma)}$. Hence, $C_{\mathrm{k}, 3}^{(\sigma)}$ is generated by the ideal classes of the primes ramified in $\mathrm{k} / \mathrm{k}_{0}$. 
* If $I$ is not principal, then the ideal class $[I]$ of $I$ generates the group $C_{\mathrm{k}, 3}^{(\sigma)}$ and it is not contained in $C_{\mathrm{k}, 3}^{1-\sigma}$. We see that $s=1$ which contradicts the fact that $s$ is even.

* If $I$ is principal, and since $\mathcal{P}$ and $\mathcal{Q}$ are also principal, then $C_{\mathrm{k}, 3}^{(\sigma)}$ will be reduced to $\{1\}$, which contradict the fact that $\left|C_{k, 3}^{(\sigma)}\right|=3$.

Hence, the prime ideal $\mathcal{P}$ is not principal.

$3) \Rightarrow 1)$ : We note that $\mathcal{P} \mathcal{Q}=(\sqrt[3]{p})$ is a principal ideal. Assume that $\mathcal{P}$ is not principal, then the prime ideal $\mathcal{Q}=\mathcal{P}^{\tau}$ is also not principal. Then $\mathcal{P} \mathcal{Q}^{2}$ is also not principal and the ideal class of $\mathcal{P} \mathcal{Q}^{2}$ generates the group $C_{\mathrm{k}, 3}^{(\sigma)}$. In addition, we have $\left(\mathcal{P} \mathcal{Q}^{2}\right)^{\tau}=\mathcal{Q P}^{2}$ which implies that $\left[\mathcal{P} \mathcal{Q}^{2}\right]^{\tau}=\left[\mathcal{Q P}^{2}\right]=\left[\mathcal{P} \mathcal{Q}^{2}\right]^{-1}$. Thus, $\left[\mathcal{P} \mathcal{Q}^{2}\right] \in\left(C_{\mathrm{k}, 3}^{(\sigma)}\right)^{-}$. According to [11, § 2, Lemma 2.1, p. 53], $C_{\mathrm{k}, 3}^{(\sigma)} \simeq\left(C_{\mathrm{k}, 3}^{(\sigma)}\right)^{+} \times\left(C_{\mathrm{k}, 3}^{(\sigma)}\right)^{-}$. Since $p \equiv 1(\bmod 3)$, then by Lemma $2.4,\left|C_{\mathrm{k}, 3}^{(\sigma)}\right|=3$, and as $\left[\mathcal{P} \mathcal{Q}^{2}\right] \in\left(C_{\mathrm{k}, 3}^{(\sigma)}\right)^{-}$, we obtain:

$$
\left|\left(C_{\mathrm{k}, 3}^{(\sigma)}\right)^{+}\right|=1 \text { and }\left|\left(C_{\mathrm{k}, 3}^{(\sigma)}\right)^{-}\right|=3 .
$$

We see that the integer $s$ is even, so the class number $h_{\mathrm{k}, 3}$ is a square. Hence $u=3$.

Lemma 2.8. Let $\mathrm{k}=\mathbb{Q}\left(\sqrt[3]{p}, \zeta_{3}\right)$, where $p$ is a prime number such that $p \equiv 4$ or $7(\bmod 9)$. Let $u$ be the index of subfield units defined as above. Then:

$$
\left(\frac{3}{p}\right)_{3} \neq 1 \Leftrightarrow\left(3 \|\left|C_{L, 3}\right| \text { and } u=3\right),
$$

where $(\dot{\bar{p}})_{3}$ is the cubic residue symbol.

Proof. Lemma 2.8 is due to Ismaili and El Mesaoudi for $e=0$ in assertion (1) of [18, Thm. 3.2, p. 104].

\subsection{Final Proof of Conjecture 1.1}

Let $L=\mathbb{Q}(\sqrt[3]{p})$ be a pure cubic field, where $p$ is a prime number such that $p \equiv 1(\bmod 3)$, and let $\mathrm{k}=\mathbb{Q}\left(\sqrt[3]{p}, \zeta_{3}\right)$ be its normal closure. Let $C_{L, 3}\left(\right.$ resp. $\left.C_{\mathrm{k}, 3}\right)$ be the 3-component of the class group of $L$ ( resp k).

1) It is clear that $C_{L, 3}$ is a cyclic group when $s=1$. Assume that $s \geq 2$. From the proof of Lemma 2.5, $C_{\mathrm{k}, 3}^{+}$and $C_{\mathrm{k}, 3}^{-}$are two non-trivial groups, and according to Lemma 2.5 we deduce that $C_{\mathrm{k}, 3}^{+}$is a cyclic group, because otherwise the rank of $C_{\mathrm{k}, 3}$ will be greater than or equal to 3 . According to $\left[11, \S 2\right.$, Lemma 2.2 , p. 53], we conclude that $C_{L, 3}$ is a cyclic group.

Now, let us show that $p \equiv 1(\bmod 9)$ when $C_{L, 3}$ contains a cyclic subgroup of order 9 . This equivalent to the statement that if $p \equiv 4$ or $7(\bmod 9)$ then the class number of $L$ is divisible exactly by 3 .

Assume that $p \equiv 4$ or $7(\bmod 9)$ and 9 divides the class number of $L$, then $\left(\frac{3}{p}\right)_{3}=1$. In fact, if $\left(\frac{3}{p}\right)_{3} \neq 1$, then from the proof of Lemma 2.8 the class number of $L$ is divisible exactly by 3 which is absurd.

Since $p=\pi_{1} \pi_{2}$, where $\pi_{1}$ and $\pi_{2}$ are two primes of $\mathrm{k}_{0}$ such that $\pi_{2}=\pi_{1}^{\tau}$, then the equations $3 \equiv X^{3}\left(\bmod \pi_{1}\right)$ and $3 \equiv X^{3}\left(\bmod \pi_{2}\right)$ are solvable in $\mathcal{O}_{\mathrm{k}_{0}}$ (cf. [17, § 9, Section 3, prop. 9.3.3, 
p.112]), so 3 is a cubic residue modulo $\pi_{1}$ and 3 is a cubic residue modulo $\pi_{2}$. According to [17, $\S$ 9, Section 1, prop. 9.1.4, p.110], we have $3=-\zeta_{3}^{2} \lambda^{2}$, where $\lambda=1-\zeta_{3}$ is a prime of $\mathrm{k}_{0}$, thus:

$$
\left(\frac{-\zeta_{3}^{2} \lambda^{2}}{\pi_{1}}\right)_{3}=\left(\frac{-\zeta_{3}^{2} \lambda^{2}}{\pi_{2}}\right)_{3}=1
$$

Since -1 is a norm in $\mathrm{k}_{0}\left(\sqrt[3]{\pi_{1}}\right) / \mathrm{k}_{0}$ and a norm in $\mathrm{k}_{0}\left(\sqrt[3]{\pi_{2}}\right) / \mathrm{k}_{0}$, then

$$
\left(\frac{-1}{\pi_{1}}\right)_{3}=\left(\frac{-1}{\pi_{2}}\right)_{3}=1
$$

so

$$
\left(\frac{\zeta_{3}^{2} \lambda^{2}}{\pi_{1}}\right)_{3}=\left(\frac{\zeta_{3}^{2} \lambda^{2}}{\pi_{2}}\right)_{3}=1
$$

Hence

$$
\left(\frac{\zeta_{3} \lambda}{\pi_{1}}\right)_{3}=\left(\frac{\zeta_{3} \lambda}{\pi_{2}}\right)_{3}=1
$$

The fields $\mathrm{k}_{0}(\sqrt[3]{\lambda})$ and $\mathrm{k}_{0}\left(\sqrt[3]{\zeta_{3}}\right)$ are different. Put $F_{1}=\mathrm{k}_{0}\left(\sqrt[3]{\zeta_{3}}\right)$ and $F_{2}=\mathrm{k}_{0}(\sqrt[3]{\lambda})$. We have $F_{1} \neq F_{2}$, and $F_{1} \cap F_{2}=\mathrm{k}_{0}$. Let $F=F_{1} F_{2}=\mathrm{k}_{0}\left(\sqrt[3]{\zeta_{3}}, \sqrt[3]{\lambda}\right)$. Then in the subfield diagram

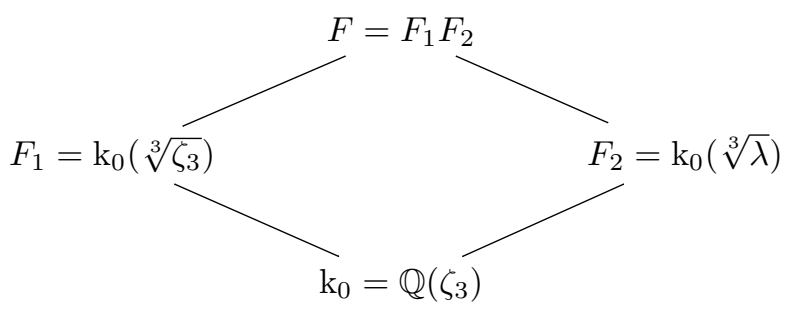

we have $\operatorname{Gal}\left(F_{1} / \mathrm{k}_{0}\right)$ and $\operatorname{Gal}\left(F_{2} / \mathrm{k}_{0}\right)$ are cyclic of order 3 , and $\operatorname{Gal}\left(F / \mathrm{k}_{0}\right)$ is abelian. Since $\pi_{1}$ is not ramified in $F_{1}$ and $F_{2}$, then $\pi_{1}$ is also not ramified in $F$. We will calculate the Artin symbol $\left(\frac{F / \mathrm{k}_{0}}{\pi_{1}}\right)$.

On the one hand:

$$
\begin{aligned}
\left(\frac{F / \mathrm{k}_{0}}{\pi_{1}}\right) & =\left(\frac{F_{1} F_{2} / \mathrm{k}_{0}}{\left(\pi_{1}\right)}\right) \\
& =\left(\frac{F_{1} / \mathrm{k}_{0}}{\left(\pi_{1}\right)}\right)\left(\frac{F_{2} / \mathrm{k}_{0}}{\left(\pi_{1}\right)}\right) \\
& =\left(\frac{\mathrm{k}_{0}\left(\sqrt[3]{\zeta_{3}}\right) / \mathrm{k}_{0}}{\left(\pi_{1}\right)}\right)\left(\frac{\mathrm{k}_{0}(\sqrt[3]{\lambda}) / \mathrm{k}_{0}}{\left(\pi_{1}\right)}\right) \\
& =\left(\frac{\zeta_{3}}{\pi_{1}}\right)_{3}\left(\frac{\lambda}{\pi_{1}}\right)_{3} \\
& =\left(\frac{\zeta_{3} \lambda}{\pi_{1}}\right)_{3} \\
& =1 .
\end{aligned}
$$


On the other hand, since $\mathrm{k}_{0}\left(\sqrt[3]{\zeta_{3}}\right)=\mathrm{k}_{0}\left(\sqrt[3]{\zeta_{3}^{2}}\right)$, we get:

$$
\begin{aligned}
\left(\frac{F / \mathrm{k}_{0}}{\pi_{1}}\right) & =\left(\frac{F_{1} F_{2} / \mathrm{k}_{0}}{\left(\pi_{1}\right)}\right) \\
& =\left(\frac{F_{1} / \mathrm{k}_{0}}{\left(\pi_{1}\right)}\right)\left(\frac{F_{2} / \mathrm{k}_{0}}{\left(\pi_{1}\right)}\right) \\
& =\left(\frac{\mathrm{k}_{0}\left(\sqrt[3]{\zeta_{3}^{2}}\right) / \mathrm{k}_{0}}{\left(\pi_{1}\right)}\right)\left(\frac{\mathrm{k}_{0}(\sqrt[3]{\lambda}) / \mathrm{k}_{0}}{\left(\pi_{1}\right)}\right) \\
& =\left(\frac{\zeta_{3}^{2}}{\pi_{1}}\right)_{3}\left(\frac{\lambda}{\pi_{1}}\right)_{3} \\
& =\left(\frac{\zeta_{3}^{2} \lambda}{\pi_{1}}\right)_{3}
\end{aligned}
$$

We see that $\left(\frac{\zeta_{3}^{2} \lambda}{\pi_{1}}\right)_{3}=1$, and since $\left(\frac{\zeta_{3} \lambda}{\pi_{1}}\right)_{3}=1$, we conclude that

$$
\left(\frac{\zeta_{3}}{\pi_{1}}\right)_{3}=1
$$

However, since $\pi_{2}$ is not ramified in $F_{1}$ and $F_{2}$, then $\pi_{2}$ is also not ramified in $F$. As above, we calculate the Artin symbol $\left(\frac{F / \mathrm{k}_{0}}{\pi_{2}}\right)$, we obtain $\left(\frac{\zeta_{3}^{2} \lambda}{\pi_{2}}\right)_{3}=1$, and since $\left(\frac{\zeta_{3} \lambda}{\pi_{2}}\right)_{3}=1$, we get

$$
\left(\frac{\zeta_{3}}{\pi_{2}}\right)_{3}=1
$$

From the cubic symbols (4) and (5) we conclude according to Lemma 2.2 that $\left(\frac{\zeta_{3}}{p}\right)_{3}=1$, so by Lemma 2.3 we obtain $p \equiv 1(\bmod 9)$ which contradicts the hypothesis that $p \equiv 4$ or $7(\bmod 9)$. Hence, if $p \equiv 4$ or $7(\bmod 9)$ then the class number of $L$ is divisible exactly by 3 .

2) Let $p$ be a prime number such that $p \equiv 4$ or $7(\bmod 9)$.

- If $\left(\frac{3}{p}\right)_{3} \neq 1$, then according to Lemma 2.8 we get $\left|C_{L, 3}\right|=3$ and $u=1$, and by Lemma 2.1 we deduce that $C_{\mathrm{k}, 3} \simeq \mathbb{Z} / 3 \mathbb{Z}$.

- If $\left(\frac{3}{p}\right)_{3}=1$, then by Lemma 2.8 we have either 3 is not divide $\left|C_{L, 3}\right|$ or $u=3$. Assume that 3 is not divide $\left|C_{L, 3}\right|$, then according to $[16, \S 1$, Thm, p. 8$]$ the prime $p$ is congruent to $-1(\bmod 3)$, which is a contradiction because $p \equiv 4$ or $7(\bmod 9)$. Hence, we have necessary $u=3$ and 3 divide $\left|C_{L, 3}\right|$, then $\left|C_{L, 3}\right|=3$ from assertion 1) above. By Lemma 2.1 we deduce that $C_{\mathrm{k}, 3} \simeq \mathbb{Z} / 3 \mathbb{Z} \times \mathbb{Z} / 3 \mathbb{Z}$.

3) According to Lemma 2.5, we get rank $C_{\mathrm{k}, 3} \in\{1,2\}$. On the one hand, for the prime numbers $p=271$ and $p=307$, we have $\left(\frac{3}{p}\right)_{3}=1$ and $p \equiv 1(\bmod 9)$, moreover for $p=271, \operatorname{rank} C_{\mathrm{k}, 3}=2$ and for $p=307$, rank $C_{\mathrm{k}, 3}=1$.

On the other hand, the primes $p=379$ and $p=487$ satisfy $\left(\frac{3}{p}\right)_{3} \neq 1$ and $p \equiv 1(\bmod 9)$, moreover for $p=379$, rank $C_{\mathrm{k}, 3}=1$ and for $p=487$, rank $C_{\mathrm{k}, 3}=2$.

This shows that, if $p \equiv 1(\bmod 9)$, then rank $C_{\mathrm{k}, 3} \in\{1,2\}$, independently of the value of $\left(\frac{3}{p}\right)_{3}$. 
From Lemmas 2.7 and 2.8, we propose the following Corollary and Proposition:

Corollary 2.0.1. Let $\mathrm{k}=\mathbb{Q}\left(\sqrt[3]{p}, \zeta_{3}\right)$, where $p$ is a prime number such that $p \equiv 4$ or $7(\bmod 9)$. Let $u$ be the index of subfield units defined as above. Then:

$$
\left(\frac{3}{p}\right)_{3} \neq 1 \Leftrightarrow u=3
$$

where $(\dot{\bar{p}})_{3}$ is the cubic residue symbol.

Proposition 2.1. Let $\mathrm{k}=\mathbb{Q}\left(\sqrt[3]{p}, \zeta_{3}\right)$, where $p$ is a prime number such that $p \equiv 4$ or $7(\bmod 9)$. Let $I, \mathcal{P}$ and $\mathcal{Q}$ be the prime ideals defined in the proof of Lemma 2.7. Then:

(i) The unique prime ideal $\mathcal{P}_{0}$ above $p$ in $\mathbb{Q}(\sqrt[3]{p})$ is principal independently of the value of $\left(\frac{3}{p}\right)_{3}$.

(ii) $\left(\frac{3}{p}\right)_{3}=1$ if and only if $I$ is principal and $\mathcal{P}$ (resp. $\mathcal{Q}$ ) is not principal.

(iii) $\left(\frac{3}{p}\right)_{3} \neq 1$ if and only if $I$ is not principal and $\mathcal{P}$ (resp. $\mathcal{Q}$ ) is principal.

where $(\dot{\bar{p}})_{3}$ is the cubic residue symbol.

The Proposition 2.1 will be underpinned by numerical examples obtained with the computational number theory system PARI [23] in $\S 3$.

Proof. Since $p \equiv 1(\bmod 3)$, then according to [17, $\S 9$, Section 1, prop. 9.1.4, p.110], $p=\pi_{1} \pi_{2}$, where $\pi_{1}$ and $\pi_{2}$ are two primes of $\mathrm{k}_{0}$ such that $\pi_{2}=\pi_{1}^{\tau}$ and $\pi_{1} \equiv \pi_{2} \equiv 1\left(\bmod 3 \mathcal{O}_{\mathrm{k}_{0}}\right)$. As $p \equiv 4$ or $7(\bmod 9)$, then 3 is ramified in $L$ by $\left[9, \S 4\right.$, pp. 51-55], so the primes ramified in $\mathrm{k} / \mathrm{k}_{0}$ are $\left(1-\zeta_{3}\right), \pi_{1}$ and $\pi_{2}$. Put $\left(\pi_{1}\right)=\mathcal{P}^{3},\left(\pi_{2}\right)=\mathcal{Q}^{3}$ and $\left(1-\zeta_{3}\right)=I^{3}$.

The fact that $p \equiv 4$ or $7(\bmod 9)$ implies that $S_{\mathrm{k}, 3}^{(\sigma)}=C_{\mathrm{k}, 3}^{(\sigma)}$, where $S_{\mathrm{k}, 3}^{(\sigma)}$ and $C_{\mathrm{k}, 3}^{(\sigma)}$ are defined in the proof of Lemma 2.7. Then $C_{\mathrm{k}, 3}^{(\sigma)}$ is generated by the ideal classes of the primes ramified in $\mathrm{k} / \mathrm{k}_{0}$.

(i) Let $\mathcal{P}_{0}$ be the unique prime ideal above $p$ in $\mathbb{Q}(\sqrt[3]{p})$, we have $p \mathcal{O}_{L}=\mathcal{P}_{0}^{3}$, and since $p \mathcal{O}_{k}=\mathcal{P}^{3} \mathcal{Q}^{3}$, then the ideal $\mathcal{P}_{0}=\mathcal{P} \mathcal{Q}=(\sqrt[3]{p})$ is principal.

(ii) Assume that $\left(\frac{3}{p}\right)_{3}=1$, then according to Corollary 2.0.1 we have $u=3$, and by Lemma 2.7 the prime ideal $\mathcal{P}$ is not principal, so $\mathcal{Q}=\mathcal{P}^{\tau}$ is also not principal. Since $\left|C_{k, 3}^{(\sigma)}\right|=3$ by Lemma 2.4 , then $I$ is principal.

(iii) Reasoning as in $(i i)$. Assume that $\left(\frac{3}{p}\right)_{3} \neq 1$, then according to Corollary 2.0.1 we have $u=3$, and by Lemma 2.7 the ideal $\mathcal{P}$ is principal, so $\mathcal{Q}=\mathcal{P}^{\tau}$ is also principal. Since $\left|C_{k, 3}^{(\sigma)}\right|=3$ by Lemma 2.4, then $I$ is not principal.

Remark 2.1. Let $L=\mathbb{Q}(\sqrt[3]{d})$, where $d>1$ is a cube-free integer, let $\mathrm{k}=\mathbb{Q}\left(\sqrt[3]{d}, \zeta_{3}\right)$ be the normal closure of the pure cubic field $L$ and $C_{L, 3}$ (resp. $C_{\mathrm{k}, 3}$ ) be the 3-component of the class group of $L$ (resp. k).

1) If $\left|C_{L, 3}\right|=3$, then rank $C_{\mathrm{k}, 3} \leq 2$.

2) If $\left|C_{L, 3}\right|=9$, then rank $C_{\mathrm{k}, 3} \leq 3$ if $u=1$, and rank $C_{\mathrm{k}, 3} \leq 4$ otherwise.

3) If $d=p$ or $p^{2}$, with $p$ is a prime number such that $p \equiv 1(\bmod 9)$, and if 9 divide exactly the class number of $\mathbb{Q}(\sqrt[3]{p})$ and $u=1$, then according to $[2, \S 1$, Theorem 1.1, p. 1], the 3 -class group of $\mathbb{Q}\left(\sqrt[3]{p}, \zeta_{3}\right)$ is of type $(9,3)$. Furthermore, if 3 is not residue cubic modulo $p$, then a generators of 3-class group of $\mathbb{Q}\left(\sqrt[3]{p}, \zeta_{3}\right)$ can be deduced by $[3, \S 3$, Theorem 3.2 , p. 10]. 


\section{Appendix}

\subsection{Illustrations of Conjecture 1.1}

Let $p \equiv 1(\bmod 3)$ be a prime, $L=\mathbb{Q}(\sqrt[3]{p})$, and $\mathrm{k}=\mathbb{Q}\left(\sqrt[3]{p}, \zeta_{3}\right)$. Let $u$ be the index of subfield units defined as above, $C_{L, 3}$ (resp. $C_{\mathrm{k}, 3}$ ) be the 3 -class group of $L$ (resp. k).

Table 1: Some numerical examples for Conjecture 1.1.

\begin{tabular}{|c|c|c|c|c|c|}
\hline$p$ & $p(\bmod 9)$ & $u$ & $\left(\frac{3}{p}\right)_{3}$ & $C_{L, 3}$ & rank $C_{\mathrm{k}, 3}$ \\
\hline 199 & 1 & 1 & & {$[9]$} & 2 \\
211 & 4 & 1 & $\neq 1$ & {$[3]$} & 1 \\
223 & 7 & 1 & $\neq 1$ & {$[3]$} & 1 \\
367 & 7 & 3 & 1 & {$[3]$} & 2 \\
499 & 4 & 3 & 1 & {$[3]$} & 2 \\
541 & 1 & 3 & & {$[9]$} & 2 \\
\hline
\end{tabular}

Moroever, in Section 17 of [4, Numerical Data, p. 238], and also in the tables of [7] which give the class number of a pure cubic field, the prime numbers $p=61,67,103$, and 151, which are all congruous to 4 or $7(\bmod 9)$, verify the following properties:

i) 3 is a residue cubic modulo $p$;

ii) 3 divide exactly the class number of $L$;

iii) $u=3$;

iv) $C_{L, 3} \simeq \mathbb{Z} / 3 \mathbb{Z}$, and $C_{\mathrm{k}, 3} \simeq \mathbb{Z} / 3 \mathbb{Z} \times \mathbb{Z} / 3 \mathbb{Z}$.

\subsection{Illustrations of Proposition 2.1 and Corollary 2.0.1}

Let $L=\mathbb{Q}(\sqrt[3]{p})$, and $\mathrm{k}=\mathbb{Q}\left(\sqrt[3]{p}, \zeta_{3}\right)$, where $p$ is a prime such that $p \equiv 4$ or $7(\bmod 9)$. We put $3 \mathcal{O}_{L}=I_{0}^{3}, p \mathcal{O}_{L}=\mathcal{P}_{0}^{3}, 3 \mathcal{O}_{\mathrm{k}}=I^{6}$, and $p \mathcal{O}_{\mathrm{k}}=\mathcal{P}^{3} \mathcal{Q}^{3}$.

Table 2: Case where $p \equiv 4$ or $7(\bmod 9)$ and $\left(\frac{3}{p}\right)_{3}=1$.

\begin{tabular}{|c|c|c|c|c|c|c|c|c|}
\hline$p$ & $u$ & $\left(\frac{3}{p}\right)_{3}$ & $C_{L, 3}$ & $C_{\mathrm{k}, 3}$ & $I_{0}$ & $I$ & $\mathcal{P}_{0}$ & $\mathcal{P}$ \\
\hline 61 & 3 & 1 & {$[3]$} & {$[3,3]$} & {$[0]$} & {$[0]$} & {$[0]$} & $\neq[0]$ \\
67 & 3 & 1 & {$[3]$} & {$[3,3]$} & {$[0]$} & {$[0]$} & {$[0]$} & $\neq[0]$ \\
103 & 3 & 1 & {$[3]$} & {$[3,3]$} & {$[0]$} & {$[0]$} & {$[0]$} & $\neq[0]$ \\
151 & 3 & 1 & {$[3]$} & {$[3,3]$} & {$[0]$} & {$[0]$} & {$[0]$} & $\neq[0]$ \\
193 & 3 & 1 & {$[3]$} & {$[3,3]$} & {$[0]$} & {$[0]$} & {$[0]$} & $\neq[0]$ \\
367 & 3 & 1 & {$[3]$} & {$[3,3]$} & {$[0]$} & {$[0]$} & {$[0]$} & $\neq[0]$ \\
439 & 3 & 1 & {$[3]$} & {$[3,3]$} & {$[0]$} & {$[0]$} & {$[0]$} & $\neq[0]$ \\
499 & 3 & 1 & {$[3]$} & {$[3,3]$} & {$[0]$} & {$[0]$} & {$[0]$} & $\neq[0]$ \\
547 & 3 & 1 & {$[3]$} & {$[3,3]$} & {$[0]$} & {$[0]$} & {$[0]$} & $\neq[0]$ \\
619 & 3 & 1 & {$[3]$} & {$[3,3]$} & {$[0]$} & {$[0]$} & {$[0]$} & $\neq[0]$ \\
643 & 3 & 1 & {$[3]$} & {$[3,3]$} & {$[0]$} & {$[0]$} & {$[0]$} & $\neq[0]$ \\
661 & 3 & 1 & {$[3]$} & {$[3,3]$} & {$[0]$} & {$[0]$} & {$[0]$} & $\neq[0]$ \\
727 & 3 & 1 & {$[3]$} & {$[3,3]$} & {$[0]$} & {$[0]$} & {$[0]$} & $\neq[0]$ \\
787 & 3 & 1 & {$[3]$} & {$[3,3]$} & {$[0]$} & {$[0]$} & {$[0]$} & $\neq[0]$ \\
853 & 3 & 1 & {$[3]$} & {$[3,3]$} & {$[0]$} & {$[0]$} & {$[0]$} & $\neq[0]$ \\
967 & 3 & 1 & {$[3]$} & {$[3,3]$} & {$[0]$} & {$[0]$} & {$[0]$} & $\neq[0]$ \\
997 & 3 & 1 & {$[3]$} & {$[3,3]$} & {$[0]$} & {$[0]$} & {$[0]$} & $\neq[0]$ \\
\hline
\end{tabular}


Table 3: Case where $p \equiv 4$ or $7(\bmod 9)$ and $\left(\frac{3}{p}\right)_{3} \neq 1$.

\begin{tabular}{|c|c|c|c|c|c|c|c|c|}
\hline$p$ & $u$ & $\left(\frac{3}{p}\right)_{3}$ & $C_{L, 3}$ & $C_{\mathrm{k}, 3}$ & $I_{0}$ & $I$ & $\mathcal{P}_{0}$ & $\mathcal{P}$ \\
\hline 7 & 1 & $\neq 1$ & {$[3]$} & {$[3]$} & $\neq[0]$ & $\neq[0]$ & {$[0]$} & {$[0]$} \\
13 & 1 & $\neq 1$ & {$[3]$} & {$[3]$} & $\neq[0]$ & $\neq[0]$ & {$[0]$} & {$[0]$} \\
31 & 1 & $\neq 1$ & {$[3]$} & {$[3]$} & $\neq[0]$ & $\neq[0]$ & {$[0]$} & {$[0]$} \\
43 & 1 & $\neq 1$ & {$[3]$} & {$[3]$} & $\neq[0]$ & $\neq[0]$ & {$[0]$} & {$[0]$} \\
79 & 1 & $\neq 1$ & {$[3]$} & {$[3]$} & $\neq[0]$ & $\neq[0]$ & {$[0]$} & {$[0]$} \\
97 & 1 & $\neq 1$ & {$[3]$} & {$[3]$} & $\neq[0]$ & $\neq[0]$ & {$[0]$} & {$[0]$} \\
139 & 1 & $\neq 1$ & {$[3]$} & {$[3]$} & $\neq[0]$ & $\neq[0]$ & {$[0]$} & {$[0]$} \\
157 & 1 & $\neq 1$ & {$[3]$} & {$[3]$} & $\neq[0]$ & $\neq[0]$ & {$[0]$} & {$[0]$} \\
211 & 1 & $\neq 1$ & {$[3]$} & {$[3]$} & $\neq[0]$ & $\neq[0]$ & {$[0]$} & {$[0]$} \\
223 & 1 & $\neq 1$ & {$[3]$} & {$[3]$} & $\neq[0]$ & $\neq[0]$ & {$[0]$} & {$[0]$} \\
229 & 1 & $\neq 1$ & {$[3]$} & {$[3]$} & $\neq[0]$ & $\neq[0]$ & {$[0]$} & {$[0]$} \\
241 & 1 & $\neq 1$ & {$[3]$} & {$[3]$} & $\neq[0]$ & $\neq[0]$ & {$[0]$} & {$[0]$} \\
277 & 1 & $\neq 1$ & {$[3]$} & {$[3]$} & $\neq[0]$ & $\neq[0]$ & {$[0]$} & {$[0]$} \\
283 & 1 & $\neq 1$ & {$[3]$} & {$[3]$} & $\neq[0]$ & $\neq[0]$ & {$[0]$} & {$[0]$} \\
313 & 1 & $\neq 1$ & {$[3]$} & {$[3]$} & $\neq[0]$ & $\neq[0]$ & {$[0]$} & {$[0]$} \\
331 & 1 & $\neq 1$ & {$[3]$} & {$[3]$} & $\neq[0]$ & $\neq[0]$ & {$[0]$} & {$[0]$} \\
\hline
\end{tabular}

\section{Acknowledgements}

The authors would like to thank Professor Daniel C. Mayer who was of a great help concerning correcting the spelling mistakes and making some precious modifications that gave more value and meaning to the work.

\section{References}

[1] S. Aouissi, D. C. Mayer, M. C. Ismaili, M. Talbi, and A. Azizi, 3-rank of ambiguous class groups in cubic Kummer extensions, arXiv preprint, 2018, arXiv:1804.00767v3.

[2] S. Aouissi, M. Talbi, M. C. Ismaili and A. Azizi. Fields $\mathbb{Q}\left(\sqrt[3]{d}, \zeta_{3}\right)$ whose 3 -class group is of type $(9,3)$. arXiv preprint, 2018, arXiv:1805.04963.

[3] S. Aouissi, M. C. Ismaili, M. Talbi and A. Azizi. The generators of 3-class group of some fields of degree 6 over $\mathbb{Q}$. arXiv preprint, 2018, arXiv:1804.00692.

[4] P. Barrucand and H. Cohn, Remarks on principal factors in a relative cubic field, J. Number Theory 3 (1971), No. 2, 226-239.

[5] P. Barrucand and H. Cohn, A rational genus, class number divisibility, and unit theory for pure cubic fields, J. Number Theory 2 (1970), No. 1, 7-21.

[6] P. Barrucand, H. C. Williams and L. Baniuk, A computational technique for determining the class number of a pure cubic field, Math. Comp. 30 (1976), No. 134, 312-323.

[7] B. D. Beach, H. C. Williams, C. R. Zarnke Some computer results on units in quadratic and cubic fields, Proceeding of the twenty-fifth summer meeting of the Canadian Mathematical Congress (Lake Head Univ., Thunder Bay, Out. 1971), 609-648.

[8] R. A. Mollin, Algebraic number theory, London, UK: Chapman and Hall (1999).

[9] R. Dedekind, Über die Anzahl der Idealklassen in reinen kubischen Zahlkörpern, J. Reine Angew. Math. 121 (1900), 40-123. 
[10] F. Gerth III, On 3-class groups of cyclic cubic extensions of certain number fields, J. Number Theory 8 (1976), No. 1, 84-98.

[11] F. Gerth III, On 3-class groups of pure cubic fields, J. Reine Angew. Math 278/279 (1975), $52-62$.

[12] F. Gerth III, On 3-class groups of certain pure cubic fields, Bull. Austral. Math. Soc. 72 (2005), 471-476.

[13] G. Gras, Sur les $\ell$-classes d'idéaux des extensions non galoisiennes de $\mathbf{Q}$ de degré premier

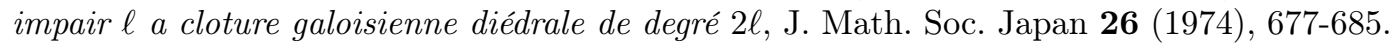

[14] H. Hasse, The class number formula of Dedekind-Meyer for simply real cubic fields, unpublished manuscript.

[15] C. S. Herz, Construction of Class Fields, Chapter VII in: A. Borel, et al., Seminar on Complex Multiplication (Inst. Advanced Study, Princeton, 1957-58), Lecture Notes in Math. 21, Springer-Verlag, New York, 1966.

[16] T.Honda, Pure cubic fields whose class numbers are multiples of three, J.Number Theory 3 (1971), No. 1, 7-12.

[17] K. Ireland and M. Rosen, A Classical Introduction to Modern Number Theory, Graduate Texts in Math. 84, Springer-Verlag, New York, 1982.

[18] M. C. Ismaili and R. El Mesaoudi, Corps cubiques purs dont le nombre de classes est exactement divisible par 3, Ann. Sci Math. Québec 28 (2004), No. 1-2, 103-112 (2005).

[19] F. Lemmermeyer, Reciprocity laws: from Euler to Eisenstein. Springer Science and Business Media, 2013.

[20] F. Lemmermeyer, Class field towers, Chapter 1, Section 1.10. Unsolved Problems, Conjecture 5 , page $44(2010)$.

[21] A. A. Markoff, Sur les nombres entiers dépendants d'une racine cubique d'un nombre entier ordinaire, Mém. Acad. Imp. Sci. St. Pétersbourg (Série VII) 38 (1892), No. 9, 1-37.

[22] C. Meyer, Die Berechnung der Klassenzahl Abelscher Zahlkörper über quadratischen Zahlkörpern, Akademie Verlag, Berlin, 1957.

[23] PARI Developer Group, PARI/GP, Version 2.9.4, Bordeaux, 2017, http://pari.math.u-bordeaux.fr.

[24] H. C. Williams, Determination of principal factors in $\mathbb{Q}(\sqrt{D})$ and $\mathbb{Q}(\sqrt[3]{D})$, Math. Comp. 38 (1982), No. 157, 261-274. 